\title{
Antibioterapia inhalada en paciente con bronquiectasias pulmonares e infección bronquial
}

\author{
Luisa Jiménez Reyes ${ }^{1}$ *, Carmen Lorenzo Martínez ${ }^{2}$, Lidia Romero Calvo ${ }^{3}$, Juan Francisco de \\ Mesa Álvarez ${ }^{4}$, Laura Lucía Checa Daimiel ${ }^{5}$, Ana Roel Conde 6, Silvia Sans Pérez ${ }^{7}$, Javier \\ Álvarez Granda ${ }^{8}$, José Javier Jareño Esteban ${ }^{9}$ \\ 1 Servicio de Medicina Interna del Hospital Central de la Defensa “Gómez Ulla” \\ 2 Servicio de Neumología del Hospital Central de la Defensa "Gómez Ulla"; ORCID id: \\ https://orcid.org/0000-0002-3236-084X \\ 3 Servicio de Medicina Interna del Hospital Central de la Defensa "Gómez Ulla"; ORCID id: \\ https://orcid.org/0000-0003-1509-2369 \\ 4 Servicio de Neumología del Hospital Central de la Defensa "Gómez Ulla"; ORCID id: \\ https://orcid.org/0000-0002-1655-9330 \\ 5 Servicio de Medicina Interna del Hospital Central de la Defensa "Gómez Ulla"; ORCID id: \\ https://orcid.org/0000-0002-8358-7024 \\ 6 Servicio de Medicina Interna del Hospital Central de la Defensa "Gómez Ulla"; ORCID id: \\ https://orcid.org/0000-0002-1318-414X \\ 7 Servicio de Neumología del Hospital Central de la Defensa "Gómez Ulla"; ORCID id: \\ https://orcid.org/0000-0002-6928-4801 \\ 8 Servicio de Medicina Interna del Hospital Central de la Defensa "Gómez Ulla"; ORCID id: \\ https://orcid.org/0000-0002-6928-4801 \\ 9 Servicio de Medicina Interna del Hospital Central de la Defensa "Gómez Ulla"; ORCID id: \\ https://orcid.org/0000-0003-2958-4487 \\ * Autor correspondencia: luisajimreyes@gmail.com; ORCID id: https://orcid.org/0000-0002-6826-2774 \\ DOI: https://doi.org/10.37536/RIECS.2021.6.2.275
}

Recibido: 04/08/2021; Aceptado: 25/10/2021; Publicado: 30/11/2021

Resumen: Las bronquiectasias pulmonares no debidas a la fibrosis quística son la tercera causa de enfermedad respiratoria crónica. Requieren un manejo integral del paciente y de sus comorbilidades. En los últimos años ha habido importantes avances con respecto al manejo y tratamiento de estos pacientes, con este trabajo pretendemos actualizar nuestro conocimiento sobre este tema a propósito de un caso. El tratamiento antibiótico inhalado es una herramienta que debemos utilizar en los pacientes con bronquiectasias pulmonares no debidas a fibrosis quística, ya que mejoran la sintomatología y la calidad de vida de los pacientes y disminuyen el número de exacerbaciones y la mortalidad.

Palabras Clave: Bronquiectasias, Infección bronquial crónica, Tomografía computarizada, Antibiótico inhalado.

\begin{abstract}
Non cystic fibrosis bronquiectasis are the third cause of chronic respiratory illness. They require a comprehensive management of the patient and its comorbidities. In the last few years, important advances in the management and treatment of these patients have been done, with this work we intend to update our knowledge on this topic in relation to a case. Inhaled antibiotic treatment is a tool that should be used in patients with non cystic fibrosis bronquiectasis, as they improve the symptoms and life quality of this patients and decrease the number of exacerbations and mortality.
\end{abstract}

Key words: Bronchiectasis, Chronic bronchial infection, Computed tomography, Inhaled antibiotics. 


\section{Introducción}

Las bronquiectasias pulmonares (BQ) se definen como la dilatación anormal y permanente de las vías aéreas. En una primera fase, predomina la inflamación bronquial con aumento de las secreciones, produciendo obstrucción al flujo aéreo. A largo plazo, se produce destrucción y fibrosis de la pared bronquial [1].

Las BQ son la tercera causa de enfermedad respiratoria crónica, tras la EPOC y el asma, pueden ser secundarias a múltiples etiologías. La terapia antibiótica inhalada constituye un tratamiento ampliamente utilizado en pacientes con fibrosis quística (FQ) con infección bronquial crónica asociada a Pseudomonas aeruginosa. Los pacientes con BQ no asociadas a FQ con infección bronquial crónica, también se benefician de esta terapia [2].

A continuación, se presenta un caso de un paciente con BQ del adulto no FQ e infección bronquial crónica que se benefició de esta modalidad terapéutica.

\section{Caso clínico}

Varón de 78 años ex-fumador (IPA 75) diagnosticado a los 52 años de BQ quísticas bilaterales, colonizadas por múltiples microorganismos (Serratia marcensens, Staphilococcus aureus meticilin resistente, Proteus mirabilis) y con evaluación pronóstica en la escala de FACED de 9. Antecedentes de EPOC GOLD IV/D y enfermedad por reflujo gastroesofágico sintomática. Presentaba frecuentes exacerbaciones respiratorias acompañadas de tos, broncorrea purulenta $(50 \mathrm{ml})$ con disnea de reposo y ruidos pulmonares audibles. A la exploración el paciente se encontraba taquipneico con cianosis y crepitantes bilaterales en la auscultación pulmonar. Entre los hallazgos de laboratorio, se objetivó acidosis respiratoria e insuficiencia respiratoria global $(\mathrm{pH} 7,26, \mathrm{pCO} 284,7 \mathrm{mmHg}$, pO2 52,9 mmHg, HCO3- $36 \mathrm{mmol} / \mathrm{l}$ ) sin leucocitosis, ni aumento de los reactantes de fase aguda (8720 leucocitos con $62 \%$ neutrófilos, $23 \%$ linfocitos, $11 \%$ monocitos, $2 \%$ eosinófilos $0,3 \%$ basófilos, PCR $0,27 \mathrm{mg} / \mathrm{dl}$, procalcitonina $0,1 \mathrm{mg} / \mathrm{dl}$ ). Se solicitó estudio de inmunoglobulinas, alfa-1 antitripsina y test del sudor que se encontraban dentro de los límites de la normalidad.

La radiografía de tórax posterioanterior y lateral (Figura 1) mostraba una afectación en hemitórax izquierdo (LII) con presencia de múltiples formaciones de aspecto quístico, algunas de ellas presentaban ocupación y nivel hidroaéreo. El TAC de tórax Figura 2) mostraba múltiples BQ quísticas bilaterales con engrosamiento de su pared (flecha gruesa), ocupación (estrella) y nivel hidroaéreo (triángulo), hallazgos relacionados con infección bronquial asociada. Todo ello producía una alteración de la arquitectura pulmonar en el LII.

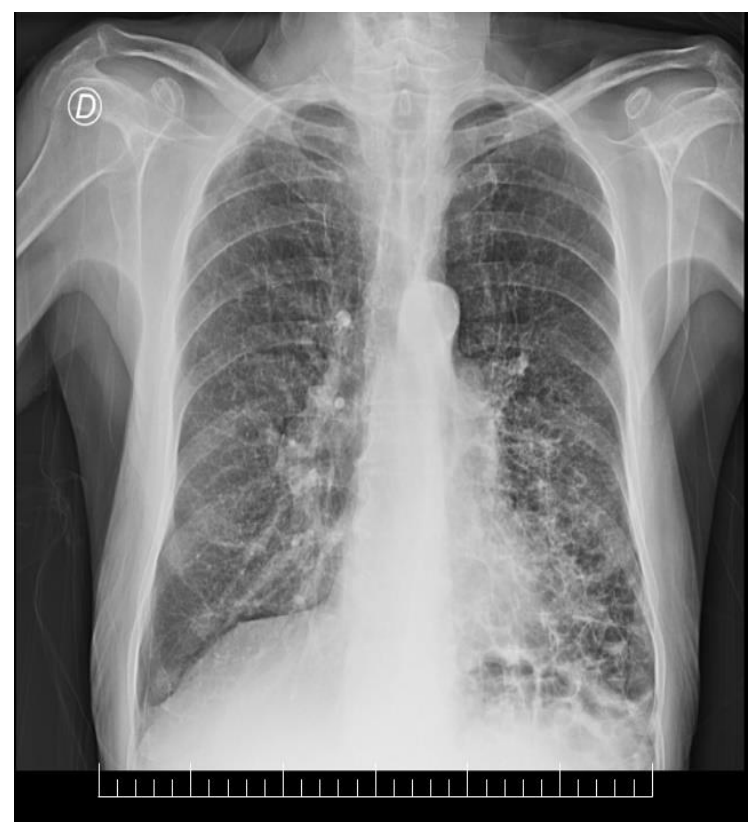

(a)

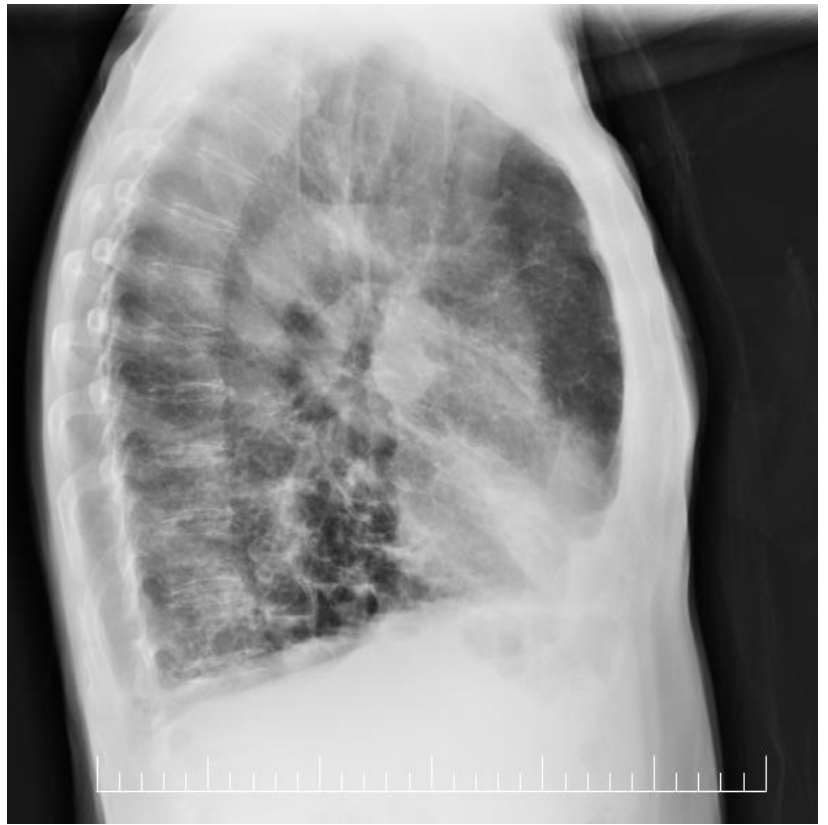

(b) 
Figura 1 Radiografía de tórax postero-anterior (a) y lateral (b)

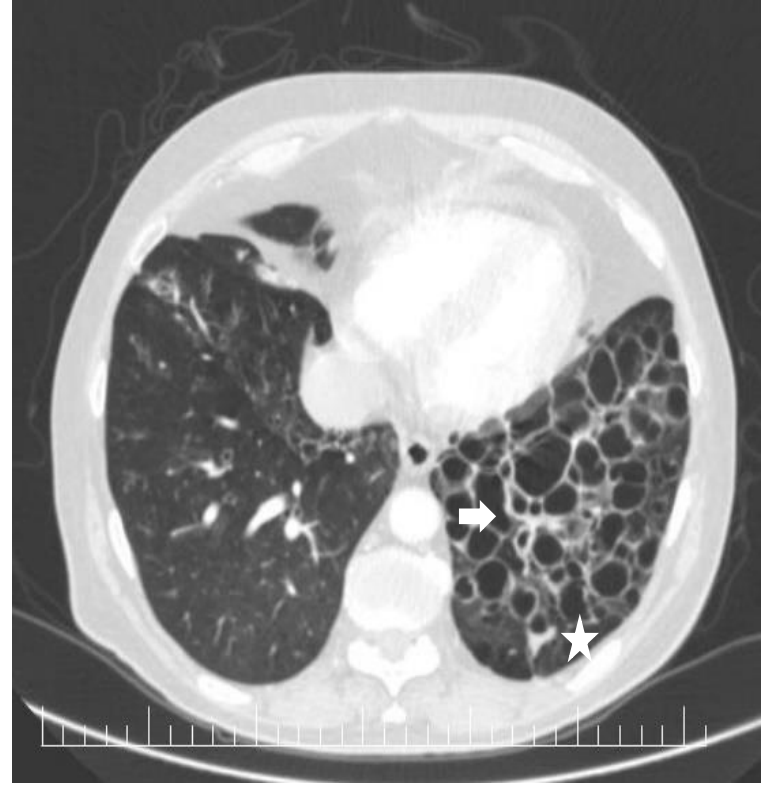

(a)

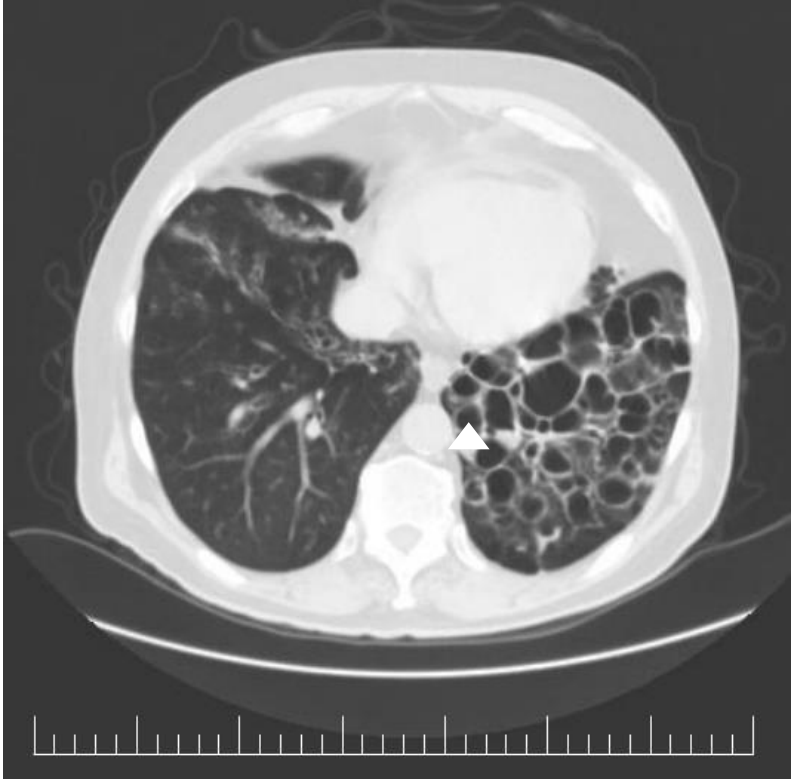

(b)

Figura 2 Cortes de TAC torácico. BQ quísticas bilaterales con engrosamiento de su pared (flecha gruesa), ocupación (estrella) (a) y nivel hidroaéreo (triángulo) (b) 
Se realizaron estudios microbiológicos del esputo para bacterias y micobacterias que confirmaron la presencia de bacilos gram negativos. Se instauró tratamiento con oxigenoterapia, fisioterapia respiratoria, broncodilatación, antibioterapia parenteral con meropenem y amikacina. Ante la persistencia de infección bronquial crónica en los cultivos microbiológicos repetidos, se inició tratamiento con colistina inhalada 1.000 .000 de unidades cada 12 horas, mediante un sistema de nebulizador de malla tipo I-neb® (Figura 3).

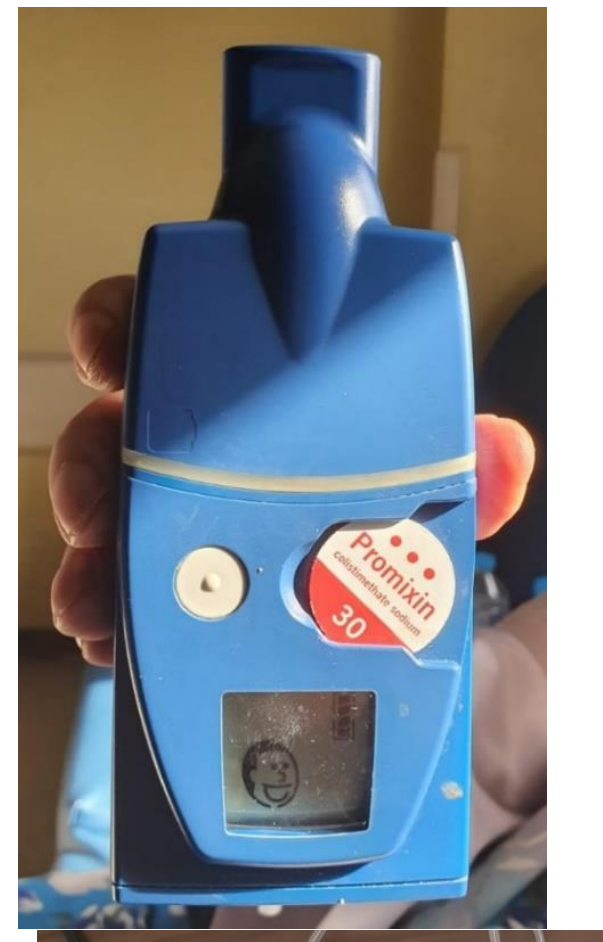

(a)

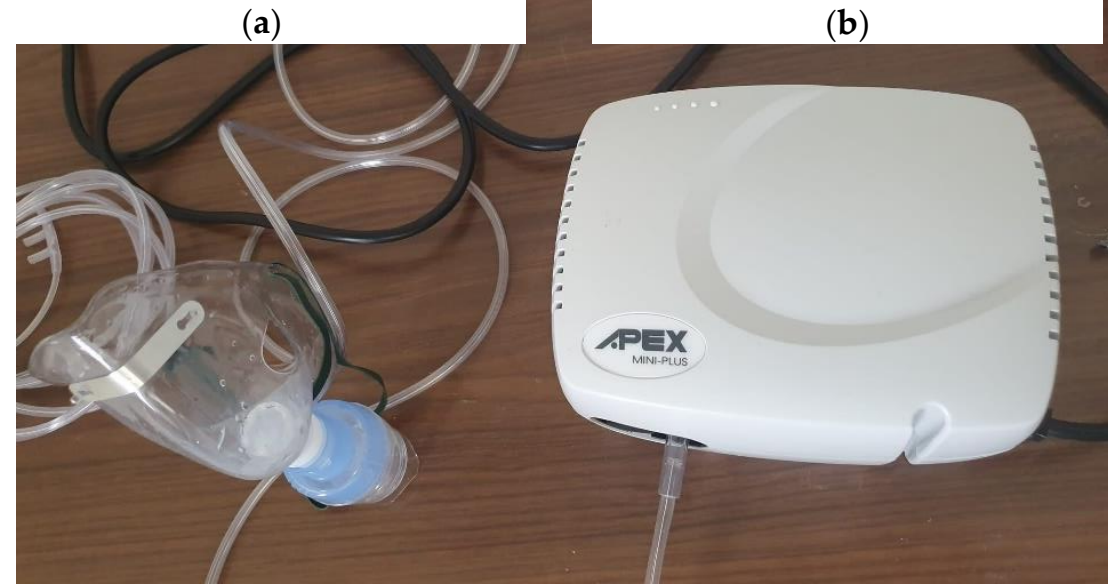

(c)

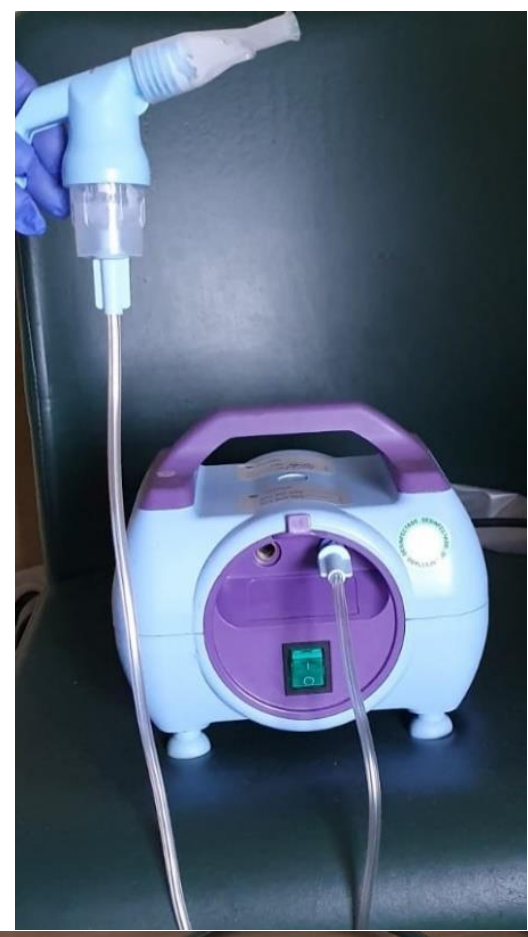

(b) 


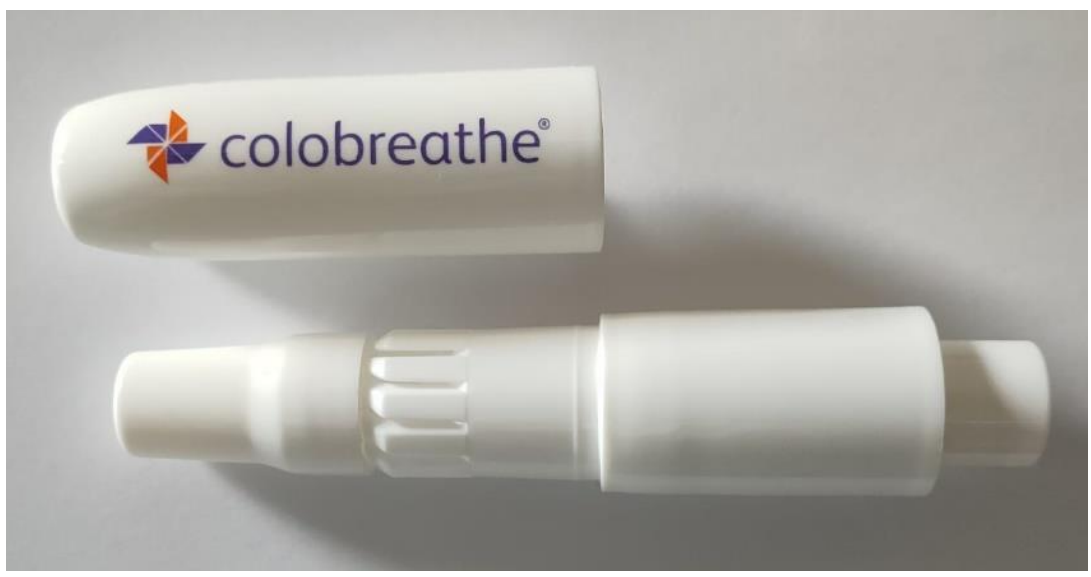

(d)

Figura 3 Sistemas de nebulización: I-neb ${ }^{\circledR}(\mathrm{a})$, e-Flow ${ }^{\circledR}(\mathrm{b})$, Nebulizador ultrasónico (c), Colobreathe (d)

Un control de TAC torácico realizado tras 6 meses de tratamiento con colistina inhalada demostró mejoría y reducción de la ocupación de las $\mathrm{BQ}$, todo ello asociado con una mejoría en la evolución clínica, en la calidad de vida y reducción de las exacerbaciones.

\section{Discusión}

El registro español de bronquiectasias presenta un mapa a través del cual podemos conocer las características de los pacientes con $\mathrm{BQ}$, la presentación y evolución de la enfermedad [3]. La prevalencia de las $\mathrm{BQ}$ no se conoce con exactitud, es posible que existan diferencias etiológicas entre los diferentes países estudiados.

En el momento del diagnóstico debemos intentar filiar la etiología, realizando estudio radiológico, analítico y genético.

Previo al desarrollo de la TAC el procedimiento diagnóstico consistía en la realización de broncografías. En la actualidad, el gold standard es la TAC ya que es un método de diagnóstico poco invasivo, indoloro, accesible y fácilmente reproducible [1]. Además, es imprescindible realizar un estudio microbiológico mediante cultivo de esputo, no solo para realizar un tratamiento antibiótico dirigido, si no porque el aislamiento microbiológico de Pseudomonas aeruginosa es factor de mal pronóstico.

Para realizar un manejo adecuado, es importante tener en cuenta diferentes variables que condicionan la calidad de vida de estos pacientes. En los últimos años se han desarrollado varias escalas multidimensionales que valoran la gravedad de esta patología, se trata de la escala FACED y la Bronchiectasis severity index (BSI). La escala FACED, publicada en el año 2014 por el grupo español de bronquiectasias (SEPAR), fue la primera escala pronóstica publicada en la literatura. En ella se tienen en cuenta variables como el valor de FEV1, la edad, la colonización crónica por Pseudomonas aeruginosa, el número de lóbulos pulmonares afectados y la disnea. Estas variables fueron identificadas como factores predictores de mortalidad a 5 años [4]. En cuanto a la escala BSI (Reino Unido), se trata de una escala pronóstica más compleja, en la que no solo se valora la mortalidad, si no también el riesgo de hospitalizaciones y exacerbaciones. Se tienen en cuenta diversos parámetros, entre los que se incluyen el IMC, las hospitalizaciones en los 2 años previos, las exacerbaciones durante el año previo y la colonización por gérmenes diferentes a Pseudomonas aeruginosa [5].

El tratamiento de las BQ en el adulto, se ha desarrollado a partir de la experiencia obtenida en el tratamiento de los pacientes con $\mathrm{FQ}$, habiéndose desarrollado en las últimas décadas diversos tratamientos por vía inhalada con múltiples dispositivos que han demostrado una mejora en la 
supervivencia, con reducción del número de exacerbaciones y de la mortalidad en los pacientes con BQ no FQ [6].

En la mayoría de los casos no es posible realizar un tratamiento específico para la causa de las $\mathrm{BQ}$, de modo que se realiza un tratamiento multidisciplinar cuyos los objetivos principales son: disminuir la obstrucción y la inflamación bronquial, favorecer el aclaramiento mucociliar y tratar la infección bronquial, además de un adecuado control de las comorbilidades. Para ello es importante el abandono del hábito tabáquico, la vacunación frente al neumococo y la gripe y adecuado soporte nutricional. Además de la fisioterapia respiratoria y el tratamiento broncodilatador de acción prolongada en los pacientes sintomáticos. En los pacientes con, al menos, 2 agudizaciones anuales a pesar del tratamiento correcto se recomienda el uso de macrólidos [6].

Otro de los pilares fundamentales del tratamiento es el manejo de la infección bronquial. La infección crónica por Pseudomonas aeruginosa se asocia a un peor pronóstico en los pacientes con $\mathrm{BQ}$, se recomienda intentar su erradicación [7]. Para recomendar su uso, los expertos se basan en el beneficio de la erradicación de este germen demostrado en múltiples estudios en paciente con FQ [8].

En la primoinfección por Pseudomonas aeruginosa, se recomienda tratamiento antibiótico oral con quinolonas durante 3 semanas, en el caso de las BQ graves FACED $\geq 6$ se añade un antibiótico inhalado desde el inicio durante 3 meses. Tras finalizar el tratamiento de la primoinfección se aconseja realizar cultivo de esputo mensual durante los 3 primeros meses y después cada 2 meses durante 1 año. Valorando reiniciar tratamiento antibiótico si hay fracaso de la erradicación [6].

En cuanto al tratamiento de la infección crónica se recomienda la utilización de antibiótico inhalado, el tratamiento se mantiene indefinidamente mientras el riesgo beneficio sea favorable. Existen varias opciones de tratamiento inhalado solo o combinado con antibióticos por vía oral, pero ninguno ha demostrado superioridad con respecto a otro. La elección de uno u otro se realizará de forma individualizada teniendo en cuenta las características del paciente y la evolución de la infección. La ventaja principal de la vía inhalada es que los antibióticos se depositan directamente en el tracto respiratorio, alcanzando mayores concentraciones en el árbol bronquial con menores efectos secundarios que por vía sistémica [6].

Diversos antibióticos son ya utilizados por vía inhalada en pacientes en FQ y BQ, como por ejemplo aztreonam lisina, etc. En múltiples ensayos clínicos se ha demostrado su eficacia, erradicando la bacteria en el esputo y previniendo agudizaciones y exacerbaciones [9].

Existen 3 tipos de sistemas de nebulización diferentes: nebulizadores ultrasónicos, tipo jet, de malla (I-neb ${ }^{\circledR}$, e-Flow ${ }^{\circledR}$ ) y de polvo seco (Colobreathe $\left.{ }^{\circledR}\right)$. La elección de uno u otro se debe realizar según las características de los pacientes. La técnica de inhalación y la preparación del dispositivo, en ocasiones son complejas, por lo que los pacientes y/o sus cuidadores necesitan un entrenamiento. Por este motivo, aunque sin duda son una excelente opción terapéutica, no se pueden utilizar todos los casos.

\section{Conclusión}

En conclusión, la antibioterapia inhalada, constituye una nueva modalidad terapéutica en la infección bronquial crónica persistente que mejora la sintomatología y la calidad de vida de los pacientes y que reduce las exacerbaciones y la mortalidad.

Conflictos de Intereses: los autores no declaran conflicto de intereses.

\section{Abreviaturas}

Las siguientes abreviaturas son usadas en este manuscrito:

BQ: Bronquiectasias

EPOC: Enfermedad pulmonar obstructiva crónica

FQ: Fibrosis Quística 
IPA: Índice Paquetes-Año

LII: Lóbulo Inferior Izquierdo

TAC: Tomografía Axial Computarizada

BSI: Bronchiectasis Severity Index

SEPAR: Sociedad Española de Neumología y Cirugía Torácica

IMC: Índice de Masa Corporal

\section{Referencias Bibliográficas}

1. Vendrell M, de Gracia J, Olveira C, Martinez-Garcia MA, Girón R, Máiz L, Cantón R, Coll R, Escribano A, Solé A. Diagnóstico y tratamiento de las bronquiectasias. SEPAR. Arch Bronconeumol. 2008;44(11):629-40.

2. Bilton D, Jones AL. Bronchiectasis: epidemiology and causes. Eur Respir Mon. 2011;52:1-10.

3. Martinez-García MA, Villa C, Dobarganes Y, Girón R, Maíz L, García-Clemente M, Sibila O, Golpe R, Rodríguez J, Barreiro E, Rodriguez JL, Menéndez R, Prados C, de la Rosa D, Olveira C; en representación del Grupo Español del Registro de Bronquiectasias (RIBRON). RIBRON: The spanish Online Bronchiectasis Registry. Characterization of the First 1912 Patients. Arch Bronconeumol. 2021;57(1):28-35.

4. Martínez-García MA, de Gracia J, Vendrell Relat M, Girón R, Máiz Carro L, de la Rosa Carrillo D, et al. Multidimensional approach to non-cystic fibrosis bronchiectasis. The FACED score. Eur Respir J. 2014;43:1357-67.

5. Chalmers JD, Goeminne P, Aliberti S, McDonnell MJ, Lonni S, Davidson J, et al. The bronchiectasis severity index. An international derivation and validation study. Am J Respir Crit Care Med. 2014;189:576-85.

6. Marínez-García MA, Máiz L, Olveira C, Girón RM, de la Rosa D, Blanco M, Cantón R, Vendrell M, Polverino E, de Gracia J, Prados C. Normativa sobre el tratamiento de las bronquiectasias en el adulto. Arch Bronconeumol. 2018;54(2):88-98.

7. Orriols R, Hernando R, Ferrer A, Terradas S, Montoro B. Eradication therapy against Pseudomonas aeruginosa in non-cystic fibrosis bronchiectasis. Respiration. 2015;90:299-305.

8. Langton Hewer SC, Smyth AR. Antibiotic strategies for eradicating Pseudomonas aeruginosa in people with cystic fibrosis. Cochrane Database Syst Rev. 2017:4:CD004197, https://doi.org/10.1002/14651858.CD004197.

9. A.M. Brodt, E. Stovold, L. Zhang. Inhaled antibiotics for stable non-cystic fibrosis bronchiectasis: A systematic review. Eur Respir J. 2014;44:382-393.

(C) 2021 por los autores; Esta obra está sujeta a la licencia de Reconocimiento 4.0 Internacional de Creative Commons. Para ver una copia de esta licencia, visite http://creativecommons.org/licenses/by-nc-nd/4.0/. 